\title{
Characterisation of tropomyosin and paramyosin as vaccine candidate molecules for the poultry red mite, Dermanyssus gallinae
}

Harry W. Wright, Kathryn Bartley, John F. Huntley and Alasdair J. Nisbet

\begin{abstract}
Background: Dermanyssus gallinae is the most economically important haematophagous ectoparasite in commercial egg laying flocks worldwide. It infests the hens during the night where it causes irritation leading to restlessness, pecking and in extreme cases anaemia and increased cannibalism. Due to an increase in the occurrence of acaricide-resistant D. gallinae populations, new control strategies are required and vaccination may offer a sustainable alternative to acaricides. In this study, recombinant forms of D. gallinae tropomyosin (Der g 10) and paramyosin (Der g 11) were produced, characterised and tested as vaccine candidate molecules.

Methods: The D. gallinae paramyosin (Der g 11) coding sequence was characterised and recombinant versions of Der g 11 and D. gallinae tropomyosin (Der g 10) were produced. Hens were immunised with the recombinant proteins and the resulting antibodies were fed to D. gallinae and mite mortality evaluated. Sections of mites were probed with anti- Der $\mathrm{g} 11$ and Der g 10 antibodies to identify the tissue distribution of these protein in D. gallinae.

Results: The entire coding sequence of Der $g 11$ was 2,622 bp encoding 874 amino acid residues.

Immunohistochemical staining of mite sections revealed that Der $\mathrm{g} 10$ and Der $\mathrm{g} 11$ were located throughout D. gallinae tissues. In phylogenetic analyses of these proteins both clustered with orthologues from tick species rather than with orthologues from astigmatid mites. Antibodies raised in hens against recombinant forms of these proteins significantly increased D. gallinae mortality, by $19 \%$ for Der $g 10(P<0.001)$ and by $23 \%$ for Der $g 11(P=0.009)$ when fed to the mites using an in vitro feeding device.

Conclusions: This study has shown that Der g 10 and Der g 11 were located ubiquitously throughout D. gallinae and that antibodies raised against recombinant versions of these proteins can be used to significantly increase D. gallinae mortality in an in vitro feeding assay. When comparing archived data for all recombinant and native proteins assessed as vaccines using this in vitro feeding assay, Der g 10 and Der g 11 ranked highly and performed better than some of the pools of native proteins.
\end{abstract}

Keywords: Dermanyssus gallinae, Paramyosin, Tropomyosin, Poultry red mite, Vaccine, Allergen

* Correspondence: Harry.Wright@moredun.ac.uk

Moredun Research Institute, Pentlands Science Park, Bush Loan, Edinburgh,

Midlothian EH26 OPZ, Scotland, UK 


\section{Background}

The poultry red mite (PRM), Dermanyssus gallinae (De Geer), is a blood-feeding ectoparasite that infests many bird species. From both an economic and welfare perspective, PRM is the most important ectoparasite affecting egg-laying hens worldwide [1, 2]. In 2005 it was estimated that $€ 130$ million was lost annually due to PRM in Europe [3] and it is likely that losses now far exceed that level. These mites reside in crevices of the furniture and machinery in the poultry house and emerge in darkness to feed, for short periods of time, on the blood of hens $[4,5]$. Heavy infestations of $D$. gallinae of more than 200,000 mites per hen can result in anaemia, irritation, restlessness, feather pecking and, in extreme cases, death and increased levels of cannibalism [5, 6]. In addition, D. gallinae is becoming an important occupational health issue for poultry workers exposed to PRM bites and allergens [7]. Spraying of poultry facilities with chemical acaricides is the most widely-used method of control [8]. However, there is an increasing incidence of resistance to acaricides in D. gallinae [9-11] and the potential for accumulation of acaricide residues in eggs and poultry meat $[12,13]$ has resulted in a need for safer alternatives for the control of PRM. Several alternative methods of control are available or are in development, for example plant or fungal extracts, essential oils and vaccination [8].

Vaccination has shown promise for the control of a number of haematophagous parasites of livestock: The commercially-available Barbervax ${ }^{\bullet}$ vaccine contains native Haemonchus contortus gut antigens and has been shown to significantly reduce clinical disease in sheep during field use in Australia (reviewed in [14]). In addition, the ectoparasite vaccines TickGARD ${ }^{\mathrm{mu}}$ and Gavac ${ }^{\mathrm{Ts}}$, were developed with the recombinant protein $\mathrm{Bm} 86$ as the protective antigen against the tropical cattle tick Rhipicephalus microplus in Australia and South America, respectively [15]. Bm86 homologues have since been fully characterised in several other tick species (Rhipicephalus annulus, $R$. decoloratus, Haemaphysalis longicornis and Hyalomma marginatum marginatum $[16,17])$ but not in other members of the Acari. To date no conserved orthologues of the Bm86 antigen have been identified in transcriptomic data of D. gallinae $[18,19]$. Therefore, other vaccine candidates are required for the development of a vaccine strategy for PRM control. One approach used for D. gallinae vaccine candidate identification has been the rational selection of antigens based upon their inferred orthology with promising vaccine candidates in other species [20]. The mite allergens tropomyosin (group 10 allergen) and paramyosin (group 11 allergen) [21] are microfilament proteins that facilitate the interaction between actin with myosin and troponin during filament assembly and contraction. Tropomyosin is ubiquitously found in eukaryotic cells and several tissue-specific isoforms exist [22, 23], whereas paramyosin is an invertebrate-specific component of the thick filament of muscles [24]. Tropomyosins are potent mite allergens [21] and both paramyosin and tropomyosin have shown promising results as vaccine candidates against a range of ecto- and endo-parasites including the trematode Schistosoma japonica, the filarioid nematode Acanthocheilonema viteae and the tick Haemaphysalis longicornis [25-28].

Tropomyosin has been characterised in a number of mite species including Metaseiulus occidentalis (94 and $97 \%$ amino acid identity to D. gallinae tropomyosin for isoforms 1 (accession XP_003745222) and 2 (accession XM_003745175), respectively), the house dust mites (HDM) Dermatophagoides pteronyssinus (85 \% identity), $D$. farinae (86\% identity) $[29,30]$ and sheep scab mites Psoroptes ovis (86 \% identity) [21]. There are also several tick species with orthologues such as $R$. microplus (89\% identity) and $H$. longicornis (88 \% identity) [31]. Recombinant tropomyosin from $\mathrm{H}$. longicornis was used to immunise rabbits and, following tick feeding on vaccinated animals, significant reductions $(P<0.05)$ in tick engorgement weights (19.4\%), oviposition (49.5\%), egg mass $(14.7 \%)$ and egg hatching rates (100 \%) were seen [27].

Paramyosin has also previously been identified as a potential vaccine candidate for parasite control: The native form of paramyosin from S. japonicum was used to immunise mice and, following challenge with the parasite, the adult worm burden in immunised mice compared to control mice was reduced by up to $86 \%$ [25]. Immunisation of BALB/c mice with a recombinant cocktail vaccine containing $S$. japonicum paramyosin fragments reduced worm burden and liver-stage eggs by up to 40 and $78 \%$, respectively following challenge [28].

The aim of the work described here was to investigate the potential of recombinant versions of $D$. gallinae tropomyosin (Der g 10) and paramyosin (Der g 11), to control the ectoparasite $D$. gallinae when used to immunise hens.

\section{Methods \\ Dermanyssus gallinae collection, extraction of total RNA and cDNA synthesis}

Mixed stage and gender D. gallinae were collected from a commercial egg production unit and were snap frozen in liquid nitrogen within $4 \mathrm{~h}$ of collection. RNA was extracted and isolated using $\mathrm{TRIZOL}^{\circ}$ reagent (Invitrogen, Carlsbad, United States) according to the manufacturer's protocol. After DNaseI treatment and subsequent purification using RNeasy spin columns (Qiagen, Hilden, Germany), mRNA was purified from total RNA using the Poly(A)Purist ${ }^{\text {tm }}$ kit (Ambion, Waltham, United States). Rapid amplification of cDNA ends (RACE)-ready cDNA was generated using the SMART ${ }^{\mathrm{Tm}}$ RACE cDNA Amplification Kit (Clontech, Paris, France) using the manufacturer's protocols. 


\section{Characterisation of the genes encoding Der $\mathrm{g} 11$ and Der $\mathbf{g} 10$}

A partial transcriptomic sequence representing the paramyosin gene Der $g 11$ was identified in a D. gallinae Roche 454 sequencing dataset [20]. 5' and 3' RACE reactions were performed to amplify the remaining sections of coding sequence. RACE products were ligated into pGEM ${ }^{-} \mathrm{T}$ Easy vector (Promega, Madison, United States) and DNA sequencing performed (Eurofins MWG Operon) to confirm the coding sequences (CDS). The full CDS of paramyosin was amplified using genespecific oligonucleotide primers (forward, 5'-TTC GGA TCC GAT GGA GGC CAT CAA GAA TAA GAT G-3' and reverse, 5'-AGT GCG GCC GCG TAT CCG GTA AGC TCG GCG AA-3') and employing RACE-ready cDNA as the template with Advantage 2 PCR polymerase (Clontech). The resulting paramyosin CDS was named Der $g 11$ in accordance with systematic allergen nomenclature [32] and deposited in the NCBI database under accession number LT555400. The CDS of the D. gallinae tropomyosin gene (Der $g$ 10, AM167555) was previously determined [31].

A homology search (Blastx) was performed using the NCBI nr database to identify homologues of Der $g 10$ and Der $g$ 11. The amino acid sequences of the top 20 hits for Der $g 11$ and Der $g 10$ were aligned using Tcoffee (Version_11.00.8cbe486) [33, 34], which generated a neighbour-joining tree drawn according to relationships inferred by the multiple sequence alignment. The neighbour joining tree was bootstrapped 1,000 times using ClustalW and viewed in the package MEGA 6.06 [35] (6140226), the degree of bootstrap was shown only if above $50 \%$.

\section{Expression and purification of Der g 10 and Der $\mathrm{g} 11$ as recombinant proteins}

The full CDS of Der $g 11$ and that of Der $g 10$ were subcloned into pET-SUMO (Invitrogen) and pET-22b(+) (Novagen, Madison, United States) vectors, respectively in frame with the vector-encoded polyhistidine tags. Plasmid DNA was transformed into Esherichia coli BL21CodonPlus $^{\oplus}$ (DE3)-RIL competent cells (Stratagene, La Jolla, United States) and recombinant protein expression induced with $1 \mathrm{mM}$ IPTG. Recombinant Der g 11 and Der g 10 were purified from inclusion bodies by nickel column affinity chromatography using HisTrap $^{\text {тм }}$ HP columns in the presence of $8 \mathrm{M}$ urea (GE Healthcare) then dialysed against $20 \mathrm{mM}$ phosphate buffer, $0.5 \mathrm{M}$ $\mathrm{NaCl}, 2 \mathrm{M}$ urea, $\mathrm{pH}$ 7.4. The Pierce $\mathrm{BCA}^{\mathrm{Tm}}$ assay (Thermo Scientific, Waltham, United States) with bovine serum albumin standards were used to estimate the protein concentration. Eluted proteins were separated by electrophoresis on a 4-12\% NuPAGE ${ }^{\odot}$ Novex Bis-Tris Gel (Invitrogen) and bands at the appropriate molecular weight for each protein were excised and analysed using MALDI-ToF spectroscopy to confirm their identity (Moredun Proteomics Unit).

\section{Generation of immunoglobulin specific for recombinant Der g 10 and Der g 11}

Female Blackrock hens were injected, into the breast muscle, with either recombinant Der g 11 or Der g 10 (2 hens for each protein). Each dose comprised $75 \mu \mathrm{g}$ of recombinant protein, $200 \mu \mathrm{g}$ of QuilA adjuvant and PBS in a final volume of $300 \mu \mathrm{l}$. Booster injections were administered 2 and 4 weeks later. Sera and eggs were collected pre-immunisation, for use as a negative controls for Western blotting and in the feeding assay, and 2 weeks after the third immunisation (week 6), when the antibody level against the target proteins was predicted to be at a maximum. IgY was purified from the egg yolks using the Eggstract kit (Promega) following the manufacturer's instructions and was stored at $-20{ }^{\circ} \mathrm{C}$. IgY concentration was determined using the Pierce $\mathrm{BCA}^{\mathrm{sm}}$ assay (Thermo Scientific). Equal amounts (3 $\mathrm{mg} / \mathrm{ml}$ ) of purified IgY from individual hens were pooled according to group and time.

All procedures were carried out in accordance with the UK Animals (Scientific Procedures) Act 1986. The ethics committee at Moredun Research Institute ratified the experimental design (08/09).

\section{Immunoblotting of $D$. gallinae extracts, recombinant Der g 10 and Der g 11}

Proteins were extracted from freshly collected $D$. gallinae and fractionated into soluble, membrane-associated, integral-membrane and insoluble proteins as previously described [36]. Each protein fraction $(\sim 3 \mu \mathrm{g}$ total protein per fraction) and recombinant protein $(3 \mu \mathrm{g})$ was loaded onto a 4-12\% NuPAGE ${ }^{\oplus}$ Novex Bis-Tris gel (Invitrogen) and then separated by electrophoresis. The proteins were electroblotted onto nitrocellulose membranes and probed with a pool of IgY previously purified from egg yolk was diluted 1:200 with Tris-buffered saline (TBS) to $\sim 30 \mu \mathrm{g} / \mathrm{ml}$ and the blot was developed as described in Bartley et al. [37].

\section{Immunolocalisation of Der $\mathrm{g} 10$ and Der $\mathrm{g} 11$ in sections of D. gallinae}

Five micron sections of $D$. gallinae were immobilised onto glass slides, using the protocol described in Bartley et al. [37]. Sections were probed with primary antibodies generated in mice against Blomia tropicalis paramyosin (Blo t 11) [38] and D. pteronyssinus tropomyosin (Der p 10) [21]. These antibodies were previously shown to cross-react with Der g 10 and Der g 11 in Western blots (data not shown).

The immunolocalisation protocol was as described in Bartley et al. [39] with the following differences: the 
primary antibodies were diluted 1:200 with TBS for the mouse monoclonal IgG anti-Blo $t$ 11, whereas the mouse polyclonal IgG anti-Der p 10 was diluted 1:1000. Bound antibodies were detected with a Rabbit anti-mouse-IgG/ HRP conjugate (Dako, Glostrup, Denmark; P0260).

\section{Effects of consuming lgY specific for Der $\mathrm{g} 10$ and Der $\mathrm{g}$ 11 on $D$. gallinae mortality}

IgY antibodies raised in hens against either Der g 10 and Der g 11 were fed to pre-conditioned D. gallinae using the in vitro blood-feeding system described in Wright et al. [36] and the mortality of mites following feeding was monitored. Briefly, three groups of mites were fed with a blood meal prepared by mixing $2 \mathrm{ml}$ fresh heparinised blood (36 USP units heparin per ml blood) obtained from D. gallinae-naive Lohmann Brown hens with $1 \mathrm{ml}(6 \mathrm{mg})$ of a pooled IgY, previously described, purified from egg yolk collected pre-immunisation (control group) or 2 weeks after the third immunisation (week 6) with Der g 10 or Der g 11 (test groups). This experiment was repeated on three occasions using 10 in vitro chambers per experimental group. Approximately 200 D. gallinae were added to each chamber and $300 \mu \mathrm{l}$ of blood containing $\operatorname{IgY}$ was added to the reservoir of each chamber. The chambers were incubated at $40{ }^{\circ} \mathrm{C}$ in darkness with a relative humidity of $75 \%$ overnight.

Mites that had fed during the overnight incubation were separated into individual wells of a 96-well microtitre plate (Greiner bio-one, Kremsmunster, Austria), sealed with AeraSeal $^{\text {Ts }}$ film (Sigma-Aldrich, St. Louis, United States; A922450EA,) and mortality was measured every $24 \mathrm{~h}$ for a 4-day period. The mite mortality data were fitted to a simple logistic regression model (SLRM), which individually compared the mortality from both test groups (Der g 10 and Der g 11-immunised) with that of the controls (immunised with adjuvant only) over the course of the experiment.

\section{Results}

Characterisation and phylogenetic relationships of Der g 10 and Der g 11

The CDS of D. gallinae Der $g 11$ (Accession number LT555400) was 2,622 bp, encoding an 874 amino acid

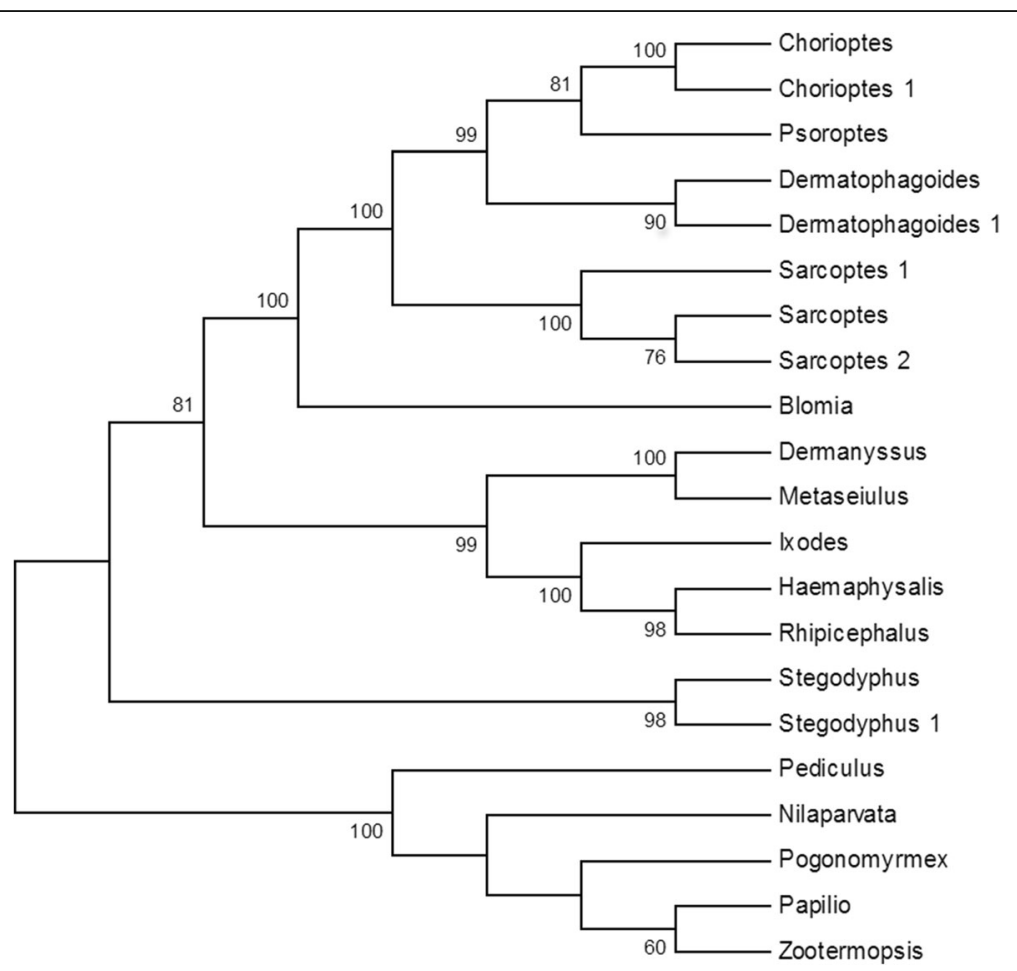

Fig. 1 Relationships between selected sequences of Der $\mathrm{g} 11$ from different invertebrate species. The neighbour-joining tree was bootstrapped 1,000 times using Clustal $X$ and the resulting tree viewed with TreeView. Each node is annotated with a figure indicating the degree of bootstrap support for each branch. Abbreviations and accession numbers are as follows: Dermanyssus (D. gallinae), Metaseiulus (M. occidentalis; XP_003744495.1), Rhipicephalus (R. (Boophilus) microplus; AAO20875.1), Haemaphysalis (H. longicornis; AFR32950.1), Ixodes (I. scapularis; XP_002407289.1), Sarcoptes (Sarcoptes scabiei; ABV54632.1), Sarcoptes 1 (S. scabiei; ACC65584.1), Dermatophagoides (D. farinae; AlO08864.1), Sarcoptes 2 (S. scabiei; AAK01181.1), Psoroptes (P. ovis; CAJ38271), Chorioptes (Chorioptes panda; ACB30406.1), Dermatophagoides 1 (D. farinae; AAO73464.1), Chorioptes 1 (C. texanus; ABK54038.1), Blomia; Blomia tropicalis (Q8MUF6.1), Stegodyphus (Stegodyphus mimosarum; KFM81308.1), Stegodyphus 1 (S. mimosarum; KFM67302.1), Pediculus (P. humanus corporis; XP_002432355.1), Zootermopsis (Zootermopsis nevadensis; KDR08790.1), Nilaparvata (Nilaparvata lugens; AGl96992.1), Pogonomyrmex (Pogonomyrmex barbatus; XP_011636912.1), Papilio (Papilio xuthus; BAM17768.1) 
sequence. The sequence with highest homology to Der g 11 was from another member of the order Mesostigmata, $M$. occidentalis with an amino acid identity of $95 \%$. The predicted protein, Der g 11, also had homologues in the tick species $R$. microplus, H. longicornis and Ixodes scapularis, with amino acid identities of 84,84 and $82 \%$, respectively whereas the amino acid identities of Der $g 11$ with the orthologous proteins from astigmatid mites were lower at $76 \%$ for Sarcoptes scabiei and $75 \%$ for P. ovis (Fig. 1).

The top two hits when the nucleotide sequence for Der g 10 was searched against the NCBI nr database using Blastx were two isoforms of tropomyosin from $M$. occidentalis with a 97 and $94 \%$ identity to Der g 10, respectively. The next two species with closest homologues were again the ticks $R$. microplus and $H$. longicornis with 89 and $88 \%$ identity, respectively. As with Der g 11, homologues from the astigmatid mite species had lower amino acid identities to Der g 10 with P. ovis and S. scabiei tropomyosins being 86 \% identical (Fig. 2).

Overall, tropomyosin and paramyosin sequences from D. gallinae were both most closely related to orthologues from species belonging to the order Mesostigmata. Phylogenetic analysis placed Der g 10 and 11 into discrete clades with the $M$. occidentalis orthologues (Figs. 1 and 2), with a stronger inferred relationship with the tick species compared to the astigmatid mites, which partitioned separately.

\section{Anatomical distribution of Der g 11 and Der g 10 in D. gallinae}

Immunoblots probed with IgY antibodies raised against the recombinant forms of Der g 10 and Der g 11 demonstrated the presence of Der g 10 in PBS-soluble, membrane-associated, integral-membrane and ureasoluble protein preparations from the mite as well as specific recognition of the recombinant bacterially-expressed Der g 10 (Fig. 3a). In contrast, Der g 11 was present only in the urea-soluble fraction of the native proteins (Fig. 3b). The recombinant form of Der g 11 was specifically bound by anti-Der g $11 \mathrm{IgY}$. The heavy and light chains of IgY were also detected in the PBS-soluble and membraneassociated proteins extracted from mites at a molecular weight of $65 \mathrm{kDa}$ and $25 \mathrm{kDa}$, respectively, on both immunoblots. Lane 11 in Fig. 3a and b show the two recombinant proteins that were used to immunise the hens.

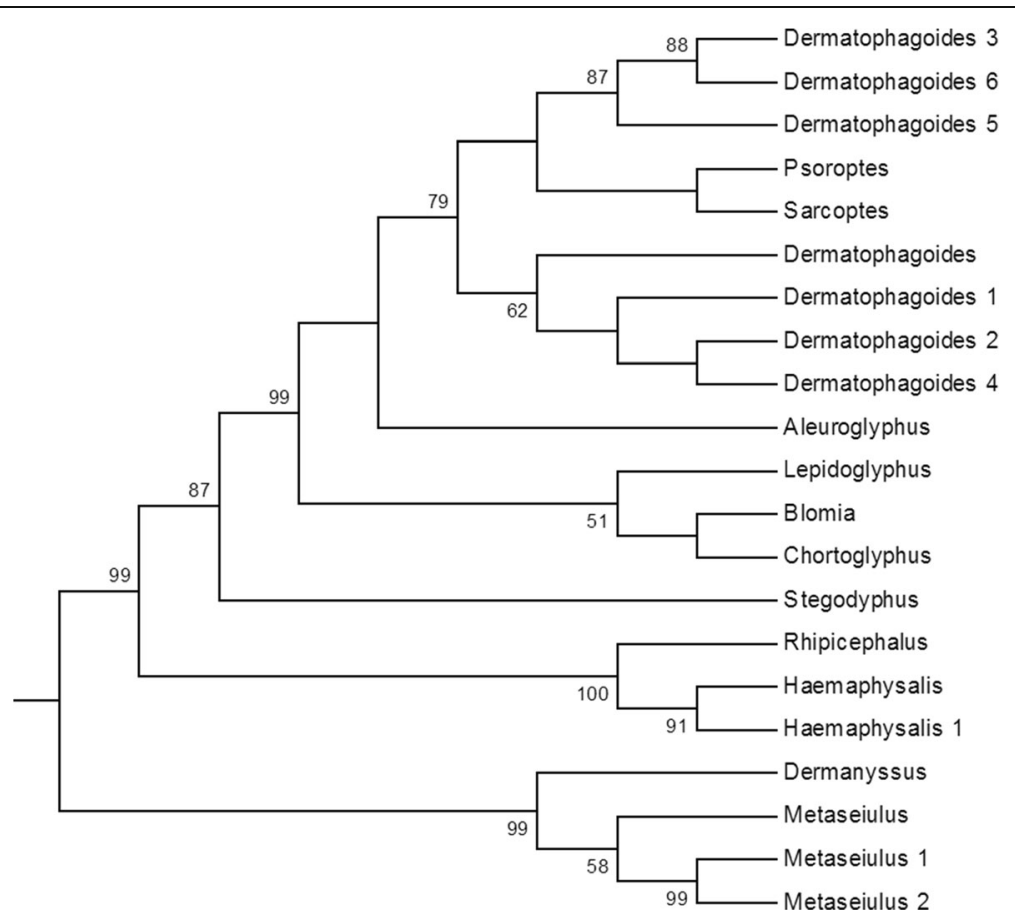

Fig. 2 Relationships between selected sequences of Der $\mathrm{g} 10$ from different invertebrate species. The neighbour-joining tree was bootstrapped 1,000 times using Clustal $X$ and the resulting tree viewed with TreeView. Each node is annotated with a figure indicating the degree of bootstrap support for each branch. Abbreviations and accession numbers are as follows: Dermanyssus (Dermanyssus gallinae; CAJ44440.1), Metaseiulus (M. occidentalis; XP_003745223.1), Metaseiulus 1 (M. occidentalis; XP_003745222.1), Rhipicephalus (R. microplus; O97162.1), Haemaphysalis (H. longicornis; Q8IT89.1), Haemaphysalis 1 (Haemaphysalis qinghaiensis; ABQ96858.1), Blomia (B. tropicalis; ABU97466.1), Lepidoglyphus (Lepidoglyphus destructor (Q9NFZ4.1)), Psoroptes (P. ovis (CAJ38272.1)), Dermatophagoides (D. farinae; BAA04557.1), Dermatophagoides 1 (D. farinae; Q23939.2), Dermatophagoides 2 (D. farinae; ABU97468.1), Dermatophagoides 3 (D. pteronyssinus; ACl32128.1), Chortoglyphus (Chortoglyphus arcuatus; AEX31649.1), Sarcoptes (S. scabiei; AFH08744.1), Stegodyphus (S. mimosarum; KFM56635.1), Dermatophagoides 4 (D. farinae; AlO08865.1), Dermatophagoides 5 (D. pteronyssinus; AAB69424.1), Dermatophagoides 6 (D. pteronyssinus; O18416.1), Aleuroglyphus (Aleuroglyphus ovatus; AAX37287.1), Metaseiulus 2 (M. occidentalis; XP_003745225.1) 


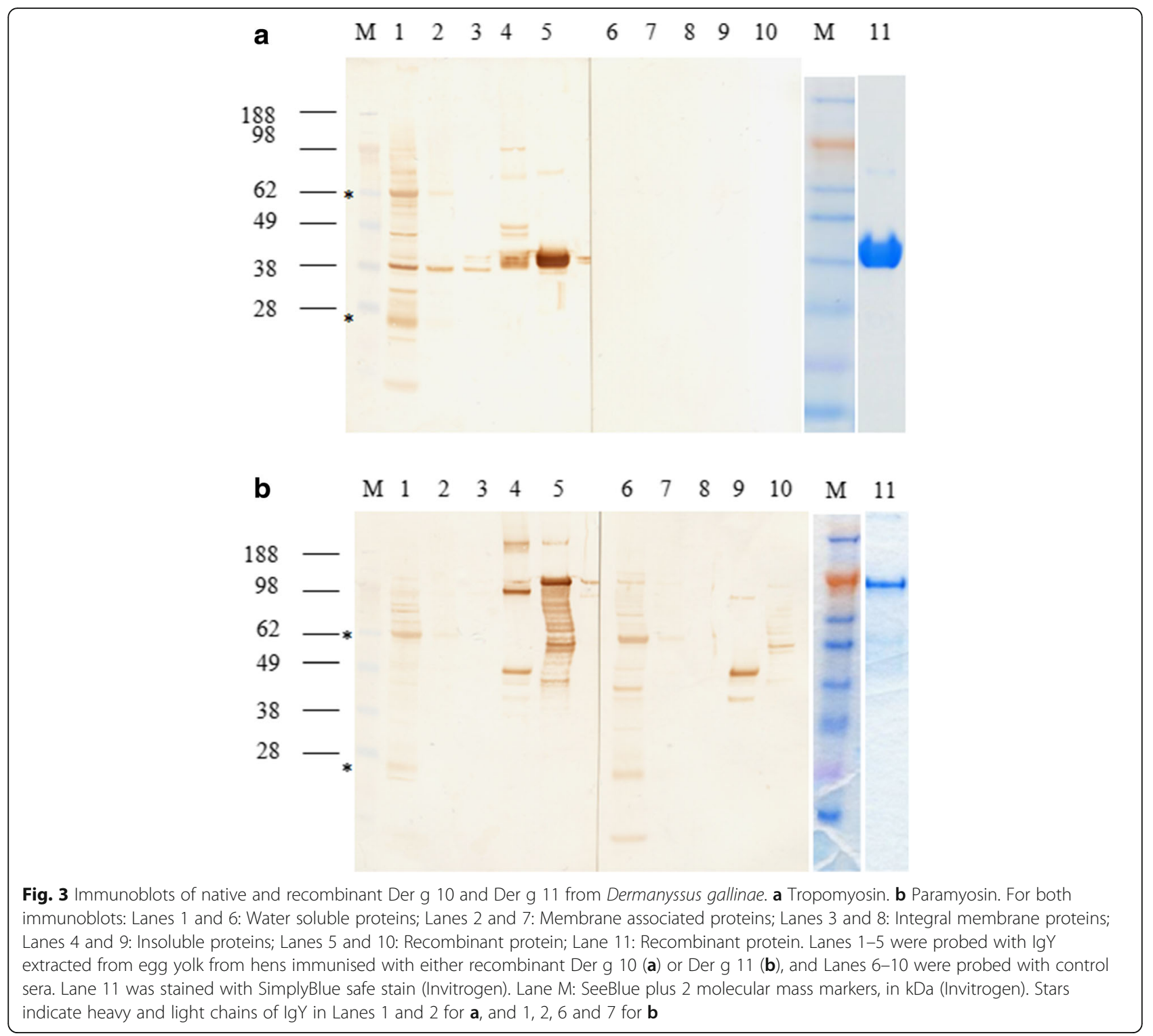

Immunolocalisation of Der g 10 and Der g 11 demonstrated specific staining of both proteins throughout the sections in multiple mite tissues, reflecting their roles in muscle movement and tissue structural integrity (Fig. 4). For both proteins, there was a high intensity of staining present in the locomotory muscle bundles compared to the staining observed in control sections [40].

\section{Effects of ingesting IgY specific for Der g 10 and Der $\mathrm{g} 11$ on $D$. gallinae mortality}

The vaccine potential of recombinant Der g 10 and Der g 11 was investigated by feeding antibodies against the proteins to $D$. gallinae in vitro. Mites were fed a blood meal enriched with $2 \mathrm{mg} / \mathrm{ml}$ of IgY generated against Der g 10, Der g 11 or IgY from nonimmunised hens to serve as a control. The majority of
D. gallinae mortality occurred within the first 2 days after feeding (Fig. 5), a mite was considered dead if it had desiccated. The mite mortality at $24 \mathrm{~h}$ was $20 \%$ ( standard error of the mean $(\mathrm{SEM})=11.3 \%)$ for Der $\mathrm{g} 11$ and $19 \%(\mathrm{SEM}=7.8 \%)$ for Der g 10, compared to $6 \%$ $(\mathrm{SEM}=3.3 \%)$ mortality observed in the control group. A further increase in mortality of $10 \%$ for Der g 11 and $7 \%$ for Der g 10 was seen from 24 to $48 \mathrm{~h}$, with a reduction in variability between mite groups (SEM reduced to 7.5 and 4.4, respectively). In comparison, the increase in mortality in the control group was lower at $3 \%(\mathrm{SEM}=3.4)$ during the same 24 to $48 \mathrm{~h}$ time period. Little mortality occurred after the $48 \mathrm{~h}$ time point for any of the groups.

The cumulative mortality of $D$. gallinae at $96 \mathrm{~h}$ after feeding on either anti-Der g 10 or anti-Der g 11 antibodies was statistically significantly higher than that of 


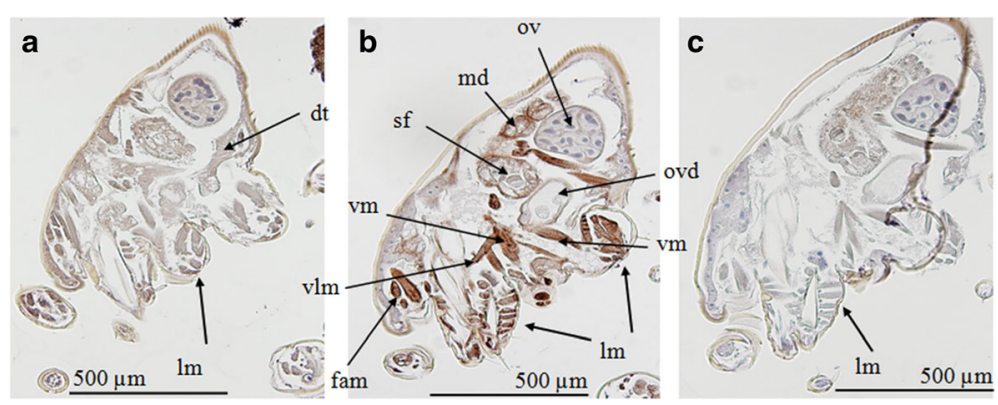

Fig. 4 Immunolocalisation of Der g 10 and Der g 11 in sections of Dermanyssus gallinae. a Der g 11. b Der g 10. c No primary antibody control slide. Abbreviations: dt, digestive tract; fam, feeding associated muscles; Im, locomotory muscle bundles; md, midgut diverticulum; ov, ovaries; ovd, oviduct; sf, sacculus foemineus; vlm, ventral longitudinal muscle; vm, ventral muscle. Brown staining represents presence of protein

the control groups $[t=4.34, d f=22, P<0.001$ for antiDer g 10 (19\% mortality); $t=2.89, d f=22, P=0.009$ for anti-Der g 11 (23\% mortality)].

\section{Discussion}

Here we have characterised $D$. gallinae allergens paramyosin (Der g 11), tropomyosin (Der g 10) and evaluated their potential as vaccine candidates. We have shown that recombinant versions of the two musclerelated proteins can generate IgY responses in hens and that when these antibodies were fed to D. gallinae, significant mortality ensued. Identifying a reliable, affordable and easy to administer control method for $D$. gallinae would improve the welfare of hens and poultry workers, increase egg production and reduce costs to the egg industry. In addition, a safe control strategy for D. gallinae that does result in potentially toxic residues in the food chain is desirable and vaccination fulfils all of those requirements. However, vaccination would not produce sterile immunity in the chickens, vaccination would be used as part of an integrated control strategy that would also include emerging techniques [8].
In recent years, several vaccine candidates have been identified in $D$. gallinae using a pragmatic approach employing successive rounds of native protein fractionation and testing and/or by selecting antigens by their inferred orthology with protective antigens from other parasite species [11, 20, 27, 31, 36, 37, 39, 41-44]. Many of the recombinant $D$. gallinae antigens or native antigen extracts previously tested for vaccine efficacy using the in vitro feeding device [36] have produced a statistically significant increase in D. gallinae mortality after one blood meal. Table 1 summarises the net increase in mite mortality $48 \mathrm{~h}$ after in vitro feeding on blood enriched with antibodies raised against the listed $D$. gallinae recombinant proteins or native protein extracts that have been tested by our group.

In comparison with the net mortality induced by other vaccine candidates listed in Table 1, Der g 11 had the fourth highest increase in mortality overall and the second highest mortality attributable to immunisation with a recombinant protein. Der g 10, which had a net increase in mortality of $18.9 \%$, was the eighth highest overall and the fifth highest recombinant protein.

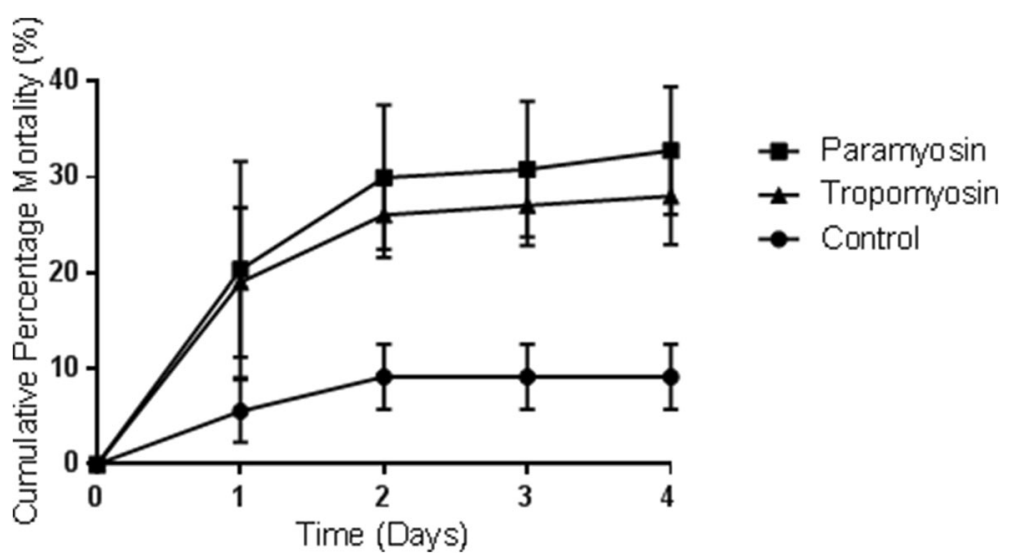

Fig. 5 Mortality of Dermanyssus gallinae. Fed heparinised chicken blood enriched with antibodies against Der g 10 or Der g 11. Mortality was monitored daily over a 4-day period following feeding. Each point represents cumulative mean percentage mortality and was derived from data recorded in three independent experiments. The error bars represent the standard error of the mean (SEM) 
Table 1 Poultry red mite mortality $48 \mathrm{~h}$ after feeding on blood enriched with antibodies. Values shown are for all recombinant proteins and native protein extracts evaluated at Moredun Research Institute thus far. Data were normalised by subtracting the percentage mortality of the mites in the control groups from the mortality of the mites in the test groups

\begin{tabular}{|c|c|c|c|}
\hline Vaccine antigen & Antigen type & $\begin{array}{l}\text { Increase in } \\
\text { percentage } \\
\text { mortality at } \\
48 \mathrm{~h} \text { after } \\
\text { feeding (\%) }\end{array}$ & Reference \\
\hline Whole SME & Native & $24.0^{*}$ & [20] \\
\hline IEX-Group-4 & Native & $23.5^{*}$ & [20] \\
\hline Deg-VIT-1 & Recombinant & $21.9^{*}$ & [20] \\
\hline Der g 11 & Recombinant & $20.1^{*}$ & Present study \\
\hline IEX-Group-1 & Native & $19.5^{*}$ & [20] \\
\hline Deg-HGP-1 & Recombinant & $18.9^{*}$ & [20] \\
\hline Deg-PUF-1 & Recombinant & $18.4^{*}$ & [20] \\
\hline Der g 10 & Recombinant & $16.5^{*}$ & Present study \\
\hline Deg-CPR-1 & Recombinant & 14.5 & [20] \\
\hline IEX-Group-3 & Native & $13.0^{*}$ & [20] \\
\hline Deg-SRP-1 & Recombinant & $12.3^{*}$ & [20] \\
\hline IEX-Group-5 & Native & $11.4^{*}$ & [20] \\
\hline PBS-soluble & Native & $10.1^{*}$ & [36] \\
\hline Dg-CatD-1 & Recombinant & $6.9^{*}$ & [39] \\
\hline Deg-ASP-1 & Recombinant & 5.6 & [20] \\
\hline Dg-HRF & Recombinant & $4.1^{*}$ & {$[37]$} \\
\hline Deg-GPD-1 & Recombinant & 4.1 & {$[20]$} \\
\hline Deg-PUF-3 & Recombinant & 3.5 & [20] \\
\hline Dg-CatL-1 & Recombinant & $2.6^{*}$ & [39] \\
\hline Membrane associated & Native & 2.2 & {$[36]$} \\
\hline Deg-PUF-2 & Recombinant & 0.6 & [20] \\
\hline Urea- soluble & Native & 0.2 & {$[36]$} \\
\hline Integral membrane & Native & -1.5 & {$[36]$} \\
\hline IEX-Group-2 & Native & -4.2 & [20] \\
\hline Deg-SRP-2 & Recombinant & -8.2 & [20] \\
\hline
\end{tabular}

*Statistically significant difference $(P<0.05)$ between mortalities in the control and antigen test groups in the original study

Therefore, Der g 11 is one of the best performing recombinant vaccine candidates tested to date in terms of inducing $D$. gallinae mortality.

When a recombinant form of tropomyosin from the astigmatid mite S. scabiei was used to immunise rabbits there was an increase in serum levels of antigen-specific IgG after immunisation. However, there was no apparent effect on mite mortality as the infested areas on the rabbits increased rapidly and mites were detected in skin scrapings from both the control groups and the vaccinated group [45]. Similarly, when a recombinant version of $H$. longicornis tropomyosin was injected into rabbits there was no impact on tick mortality following challenge but immunisation with the recombinant $H$. longicornis tropomyosin did significantly reduce the engorgement weight (150 mg compared to $186 \mathrm{mg}$ in the control; $P<0.05)$, egg laying rate (6\% compared to $56 \%$ in the controls; $P<0.05)$ and egg hatching, which was totally blocked ( $0 \%$ after vaccination) of ticks feeding on the immunised hosts [27]. These reproductionassociated indicators of vaccine efficacy were not measured in the current study but with further refinement of the in vitro feeding assay they could be incorporated into future vaccine efficacy studies.

In vaccine studies with other parasites, using paramyosin and tropomyosin as antigens, a $34 \%$ reduction in $A$. viteae adult worm burden was recorded when tropomyosin was used to immunise jirds [41] and reductions of S. japonicum burden of between 23 and $40 \%$ have been recorded when paramyosin was used to immunise mice [28]. Paramyosin from the liver fluke Chlonorchis sinensis was also used in a vaccine study where a recombinant version of the protein was used to immunise rats, which were then challenged with metacercariae. The faecal egg counts (51\% of those measured in controls) and worm burdens ( $54 \%$ of those measured in the controls) were significantly reduced by immunisation [42]. When a recombinant version of Dictyocaulus viviparus paramyosin was used to vaccinate cattle it caused a $52 \%$ average reduction in larval shedding and a $44 \%$ reduction in worm burden over two experiments [43].

These studies all used endo-parasitic organisms that are continually under pressure from the host immune system. The in vitro feeding assay used herein for an ectoparasitic organism only offers $D$. gallinae blood for a single night, which equates to one blood meal. During the course of the D. gallinae life-cycle, a mite will feed multiple times [46], which would frequently expose it to the host immune system. The percentage mortality observed could therefore increase as D. gallinae feed multiple times, potentially leading to impact on $D$. gallinae populations in a field setting. In addition, as the mortality of D. gallinae fed anti-Der g 10 and anti-Der g 11 antibodies individually was relatively low these, or other antigens, may be combined to produce a cocktail vaccine whose components could act additively or synergistically to produce a higher level of mortality (e.g. see [47]).

The mechanisms of action behind the mortality-inducing effects of ingested anti-Der g 10 and anti-Der g 11 are not yet understood but, for both proteins, antibody-binding to mite tissues may inhibit the functions of the proteins, leading to disruption of cytoskeletal function or formation [47]. Evidence has also been found that paramyosin, from Trichinella spiralis, can sequester host $\mathrm{C} 9$, reducing cell damage to the parasite from the host complement cascade, so a vaccine based on paramyosin may prevent the parasite from subverting the host immune response [48]. Both of these 
actions would ultimately cause cell death and the breakdown of D. gallinae gut. This breakdown of the gut could explain the characteristic "red leg" phenotype that can be seen in some of the dead D. gallinae observed [18].

\section{Conclusions}

The experiments described in this paper have shown that tropomyosin and paramyosin are present in D. gallinae and, when they were used as immunising antigens in hens an antibody response was generated against them, which had a statistically significantly deleterious effect on the survival of D. gallinae. Therefore, these antigens should be considered as potential vaccine candidates against $D$. gallinae, the poultry red mite.

\section{Abbreviation}

PRM: Poultry red mite

\begin{abstract}
Acknowledgments
This work was supported by a postgraduate training award to HW by the British Egg Marketing Board Trust and AN and KB were supported in this work by a DEFRA grant (award number AW 1138). The authors would like to acknowledge the continuing support of Mr. John Campbell, Glenrath Farms, United Kingdom for this project.
\end{abstract}

\section{Funding}

This work was supported by a postgraduate training award to HW by the British Egg Marketing Board Trust and AN and KB were supported in this work by a DEFRA grant (award number AW 1138).

\section{Availability of data and materials}

Data supporting the conclusions of this article are included within the article. The sequence for Der $g 11$ was submitted in the European Nucleotide Archive database under accession number LT555400.

\section{Authors' contributions}

All authors had input in designing the experiment; HW preformed the experiments with assistance from $\mathrm{KB} ; \mathrm{HW}$ drafted the manuscript with editorial input from the other authors; all authors read and approved the final version of the manuscript.

\section{Competing interests}

The authors declare that they have no competing interests.

\section{Consent for publication}

Not applicable.

\section{Ethics approval and consent to participate}

All procedures were carried out in accordance with the UK Animals (Scientific Procedures) Act 1986. The ethics committee at Moredun Research Institute ratified the experimental design (08/09).

Received: 19 May 2016 Accepted: 4 October 2016

Published online: 12 October 2016

\section{References}

1. Kim Sl, Na YE, Yi JH, Kim BS, Ahn YJ. Contact and fumigant toxicity of oriental medicinal plant extracts against Dermanyssus gallinae (Acari: Dermanyssidae). Vet Parasitol. 2007;145(3-4):377-82.

2. Roy L, Chauve C. The Genus Dermanyssus (Mesostigmata: Dermanyssidae): History and Species Characterization, Trends in Acarology. Dordrecht: Springer Science + Business Media B.V; 2009

3. Van Emous R. Wage war against the red mite! Poultry Int. 2005;44:8.

4. Axtell RC, Arends JJ. Ecology and management of arthropod pests of poultry. Annu Rev Entomol. 1990;35:101-26.

5. Kilpinen O, Roepstorff A, Permin A Norgaard-Nielsen G, Lawson LG, Simonsen HB. Influence of Dermanyssus gallinae and Ascaridia galli infections on behaviour and health of laying hens (Gallus gallus domesticus). Br Poult Sci. 2005:46(1):26-34

6. Chauve C. The poultry red mite Dermanyssus gallinae (De Geer, 1778): current situation and future prospects for control. Vet Parasitol. 1998;79(3): 239-45.

7. George DR, Finn RD, Graham KM, Mul MF, Maurer V, Moro CV, et al. Should the poultry red mite Dermanyssus gallinae be of wider concern for veterinary and medical science? Parasit Vectors. 2015;8:178.

8. Sparagano OA, George DR, Harrington DW, Giangaspero A. Significance and control of the poultry red mite, Dermanyssus gallinae. Annu Rev Entomol. 2014;59:447-66.

9. Beugnet F, Chauve C, Gauthey M, Beert L. Resistance of the red poultry mite to pyrethroids in France. Vet Rec. 1997;140(22):577-9.

10. Marangi M, Cafiero MA, Capelli G, Camarda A, Sparagano OA, Giangaspero A. Evaluation of the poultry red mite, Dermanyssus gallinae (Acari: Dermanyssidae) susceptibility to some acaricides in field populations from Italy. Exp Appl Acarology. 2009:48(1-2):11-8.

11. Bartley K, Wright HW, Bull RS, Huntley JF, Nisbet AJ. Characterisation of Dermanyssus gallinae glutathione S-transferases and their potential as acaricide detoxification proteins. Parasit Vectors. 2015;8:350.

12. Hamscher $G$, Priess $B$, Nau H. Determination of phoxim residues in eggs by using high-performance liquid chromatography diode array detection after treatment of stocked housing facilities for the poultry red mite (Dermanyssus gallinae). Anal Chim Acta. 2007;586(1-2):330-5.

13. Marangi M, Morelli V, Pati S, Camarda A, Cafiero MA, Giangaspero A. Acaricide residues in laying hens naturally infested by red mite Dermanyssus gallinae. PLoS One. 2012;7(2), e31795.

14. Nisbet AJ, Meeusen EN, González JF, Piedrafita DM. Immunobiology of Haemonchus contortus and vaccine development. Adv Parasitol. 2016;93: 353-96.

15. Rand KN, Moore T, Sriskantha A, Spring K, Tellam R, Willadsen P, et al. Cloning and expression of a protective antigen from the cattle tick Boophilus microplus. Proc Natl Acad Sci U S A. 1989;86(24):9657-61.

16. Canales M, de la Lastra JM, Naranjo V, Nijhof AM, Hope M, Jongejan F, et al. Expression of recombinant Rhipicephalus (Boophilus) microplus, R. annulatus and $R$. decoloratus Bm86 orthologs as secreted proteins in Pichia pastoris. BMC Biotechnol. 2008:8:14

17. Liao M, Zhou J, Hatta T, Umemiya R, Miyoshi T, Tsuji N, et al. Molecular characterization of Rhipicephalus (Boophilus) microplus Bm86 homologue from Haemaphysalis longicornis ticks. Vet Parasitol. 2007;146(1-2):148-57.

18. Wright HW. Identification of Vaccine Candidates Against the Poultry Red Mite, Dermanyssus Gallinae. Edinburgh: University of Edinburgh; 2011.

19. Schicht S, Qi W, Poveda L, Strube C. Whole transcriptome analysis of the poultry red mite Dermanyssus gallinae (De Geer, 1778). Parasitology. 2014; 141(3):336-46.

20. Bartley K, Wright HW, Huntley JF, Manson ED, Inglis NF, McLean K, et al. Identification and evaluation of vaccine candidate antigens from the poultry red mite (Dermanyssus gallinae). Int J Parasitol. 2015;45(13):819-30.

21. Huntley JF, Machell J, Nisbet AJ, Van den Broek A, Chua KY, Cheong N, et al. Identification of tropomyosin, paramyosin and apolipophorin/vitellogenin as three major allergens of the sheep scab mite, Psoroptes ovis. Parasite Immunol. 2004;26(8-9):335-42.

22. Perry SV. Vertebrate tropomyosin: distribution, properties and function. J Muscle Res Cell Motility. 2001;22(1):5-49.

23. Sereda MJ, Hartmann S, Lucius R. Helminths and allergy: the example of tropomyosin. Trends Parasitol. 2008;24(6):272-8.

24. Elfvin M, Levine RJ, Dewey MM. Paramyosin in invertebrate muscles. I. Identification and localization. J Cell Biol. 1976;71(1):261-72.

25. Ramirez BL, Kurtis JD, Wiest PM, Arias P, Aligui F, Acosta L, et al. Paramyosin: a candidate vaccine antigen against Schistosoma japonicum. Parasite Immunol. 1996;18(1):49-52.

26. Hartmann S, Sereda MJ, Sollwedel A, Kalinna B, Lucius R. A nematode allergen elicits protection against challenge infection under specific conditions. Vaccine. 2006:24(17):3581-90.

27. Tian M, Tian Z, Luo J, Xie J, Yin H, Zeng Q, et al. Identification of the tropomyosin (HL-Tm) in Haemaphysalis longicornis. Vet Parasitol. 2015: 207(3-4):318-23.

28. Zhang DM, Pan WQ, Qian L, Duke M, Shen LH, McManus DP. Investigation of recombinant Schistosoma japonicum paramyosin fragments for immunogenicity and vaccine efficacy in mice. Parasite Immunol. 2006;28(3): $77-84$. 
29. Asturias JA, Arilla MC, Gomez-Bayon N, Martinez A, Martinez J, Palacios R. Sequencing and high level expression in Escherichia coli of the tropomyosin allergen (Der p 10) from Dermatophagoides pteronyssinus. Biochim Biophys Acta. 1998;1397(1):27-30

30. Aki T, Kodama T, Fujikawa A, Miura K, Shigeta S, Wada T, et al. Immunochemical characterization of recombinant and native tropomyosins as a new allergen from the house dust mite, Dermatophagoides farinae. J Allergy Clin Immunol. 1995;96(1):74-83.

31. Nisbet AJ, Huntley JF, Mackellar A, Sparks N, McDevitt R. A house dust mite allergen homologue from poultry red mite Dermanyssus gallinae (De Geer). Parasite Immunol. 2006:28(8):401-5.

32. Chapman MD, Pomes A, Breiteneder H, Ferreira F. Nomenclature and structural biology of allergens. J Allergy Clin Immunol. 2007;119(2):414-20.

33. Notredame C, Higgins DG, Heringa J. T-coffee: a novel method for fast and accurate multiple sequence alignment. J Mol Biol. 2000;302(1):205-17.

34. Di Tommaso P, Moretti S, Xenarios I, Orobitg M, Montanyola A, Chang JM, et al. T-Coffee: a web server for the multiple sequence alignment of protein and RNA sequences using structural information and homology extension. Nucleic Acids Res. 2011;39(Web Server issue):W13-7.

35. Tamura K, Stecher G, Peterson D, Filipski A, Kumar S. MEGA6: Molecular Evolutionary Genetics Analysis version 6.0. Mol Biol Evol. 2013;30(12):2725-9.

36. Wright HW, Bartley K, Nisbet AJ, McDevitt RM, Sparks NH, Brocklehurst S, et al. The testing of antibodies raised against poultry red mite antigens in an in vitro feeding assay; preliminary screen for vaccine candidates. Exp Appl Acarology. 2009;48(1-2):81-91.

37. Bartley K, Nisbet AJ, Offer JE, Sparks NH, Wright HW, Huntley JF. Histamine release factor from Dermanyssus gallinae (De Geer): characterization and in vitro assessment as a protective antigen. Int J Parasitol. 2009;39(4):447-56.

38. Ramos JD, Teo AS, Ou KL, Tsai LC, Lee BW, Cheong N, et al. Comparative allergenicity studies of native and recombinant Blomia tropicalis Paramyosin (Blo t 11). Allergy. 2003;58(5):412-9.

39. Bartley K, Huntley JF, Wright HW, Nath M, Nisbet AJ. Assessment of cathepsin D and L-like proteinases of poultry red mite, Dermanyssus gallinae (De Geer), as potential vaccine antigens. Parasitology. 2012;139(6):755-65.

40. Sonenshine DE, Roe RM. Biology of Ticks. Secondth ed. Oxford: Oxford University Press; 2014.

41. Hartmann S, Adam R, Marti T, Kirsten C, Seidinger S, Lucius R. A 41-kDa antigen of the rodent filaria Acanthocheilonema viteae with homologies to tropomyosin induces host-protective immune responses. Parasitol Res. 1997; 83(4):390-3

42. Wang $X$, Chen W, Lv X, Tian Y, Men J, Zhang X, et al. Identification and characterization of paramyosin from cyst wall of metacercariae implicated protective efficacy against Clonorchis sinensis infection. PLoS One. 2012;7(3), e33703.

43. Strube C, Haake C, Sager H, Schorderet Weber S, Kaminsky R, Buschbaum S, et al. Vaccination with recombinant paramyosin against the bovine lungworm Dictyocaulus viviparus considerably reduces worm burden and larvae shedding. Parasit Vectors. 2015:8:119.

44. Harrington D, Canales M, de la Fuente J, de Luna C, Robinson K, Guy J, et al. Immunisation with recombinant proteins subolesin and Bm86 for the control of Dermanyssus gallinae in poultry. Vaccine. 2009;27(30):4056-63.

45. Zhang R, Jise Q, Zheng W, Ren Y, Nong X, Wu X, et al. Characterization and evaluation of a Sarcoptes scabiei allergen as a candidate vaccine. Parasit Vectors. 2012;5:176.

46. Kilpinen O, Mullens BA. Effect of food deprivation on response of the mite Dermanyssus gallinae, to heat. Med Vet Entomol. 2004;18(4):368-71.

47. Mizuno H, Hamada A, Shimada K, Honda H. Tropomyosin as a regulator of the sliding movement of actin filaments. Bio Systems. 2007;90(2):449-55.

48. Zhao X, Hao Y, Yang J, Gu Y, Zhu X. Mapping of the complement C9 binding domain on Trichinella spiralis paramyosin. Parasit Vectors. 2014;7:80.

\section{Submit your next manuscript to BioMed Central and we will help you at every step:}

- We accept pre-submission inquiries

- Our selector tool helps you to find the most relevant journal

- We provide round the clock customer support

- Convenient online submission

- Thorough peer review

- Inclusion in PubMed and all major indexing services

- Maximum visibility for your research

Submit your manuscript at www.biomedcentral.com/submit
Biomed Central 